\title{
Comparative Study of Antibody Avidity in Single and Multiple Vaccinated Animals Against Foot-and-Mouth Disease
}

\author{
Beenu Jain ${ }^{1 *}$, Anuj Tewari ${ }^{2}$ and Surender Kumar Kadian ${ }^{1}$ \\ ${ }^{1}$ Department of Veterinary Microbiology, College of Veterinary Sciences, Lala \\ Lajpat Rai University of Veterinary and Animal Sciences, Hisar, Haryana, India \\ ${ }^{2}$ Department of Veterinary Microbiology, College of Veterinary and Animal \\ Sciences, Govind Ballabh Pant University of Agriculture and Technology, \\ Pantnagar, Uttarakhand, India \\ *Corresponding Author: Beenu Jain, Department of Veterinary Microbiology, \\ College of Veterinary Sciences, Lala Lajpat Rai University of Veterinary and Animal \\ Sciences, Hisar, Haryana, India.
}

Received: January 13, 2021

Published: January 31, 2022

(C) All rights are reserved by Beenu Jain., et al.

\begin{abstract}
In this study, serum samples from single vaccinated and multiple vaccinated animals, against Foot-and-mouth disease (FMD) were collected from field buffaloes. Samples with titre more than 1.4 in LPBE were selected for measuring antibody avidity. The study was conducted to compare the actual status of the protective antibody generated after vaccination in single and multiple vaccinated animals. The binding affinity of these antibodies were tested using an avidity ELISA. It was found that even after multiple vaccination in some of the animals the avidity was low whereas some of the single vaccinated animals showed high avidity than the multiple vaccinated animals. We conclude that multiple vaccination with strong antibody titre may not be always a true indicator of strong protective response. Hence, regular post-vaccination monitoring of antibody avidity needs to be done where there is a high virus circulation as a measure of herd immunity.
\end{abstract}

Keywords: Avidity ELISA; Foot-and-Mouth Disease; Post-Vaccination Monitoring; Herd Immunity

\section{Abbreviations}

LPBE: Liquid Phase Blocking ELISA; AI: Avidity Index; FMD: Footand-Mouth Disease; FMDV: Foot-and Mouth Disease Virus; PVM: Post-Vaccination Monitoring

\section{Introduction}

Foot-and-mouth disease (FMD) is an economically important disease of domestic and wild cloven-hoofed animals. It is caused by the Foot-and-mouth disease virus of the family Picornaviridae. In endemic countries, the annual loss incurred by FMD outbreaks and cost of vaccination amount to US\$ 6.5 to 21 billion [1]. Additionally, FMD outbreaks in FMD free countries cause an annual loss of more than US\$ 1.5 billion [1]. FMD outbreaks in endemic countries are controlled by biannual vaccination. Both pre-vaccinated and post-vaccinated sera are collected and are subjected to Liquid Phase Blocking ELISA (LPBE) to measure and compare the protective antibody titer generated. The antibody generated after vaccination is considered to be protective and is considered to prevent future outbreaks, although, it does not provide sterile immunity.

In an endemic setting, along with the measurement of antibody titer, it is also necessary to measure the binding affinity of the antibodies with the antigen, particularly in the case of multiple vaccinated animals where the booster dose is given in every six months. This can be achieved by developing an avidity ELISA to check the strength of binding of antibodies with their corresponding antigen. The avidity of an antibody refers to the strength of its bonding with antigen and is related to the antigen-antibody site. Avidity ELISA has also been applied for the assessment of heterologous protec-

Citation: Beenu Jain., et al. "Comparative Study of Antibody Avidity in Single and Multiple Vaccinated Animals Against Foot-and-Mouth Disease". Acta Scientific Veterinary Sciences 4.2 (2022): 101-104. 
tion against FMDV in cattle [2]. Likewise, the avidity of antibody responses against FMDV and its relationship with protection has not been investigated, although the idea of its relevance in complementing quantitative assessments has been already proposed in previous reports [3-6]. There is also a gap in the knowledge about avidity of antibodies developed in pre and post vaccinated animals against FMD.

In this study we have compared the avidity of the antibodies developed in single and multiple vaccinated animals against FMD.

\section{Materials and Methods}

\section{Serum samples}

In this study, forty-seven serum samples from multiple vaccinated serum samples and forty two serum samples from single vaccinated animals were collected randomly from the field. These animals were already vaccinated by a trivalent inactivated FMDV vaccine (comprising of $0, A$ and Asia 1 antigens) by the field veterinarians. Multiple vaccinated animals are those animals which have been vaccinated more than two times by using FMDV trivalent vaccine. The serum samples were collected after consent from the animal owners. Proper history of FMD vaccination was taken for both single and multiple FMDV vaccinated animals. Only samples showing LPBE titer more than 1.4, suggestive of strong antibody titer were tested for their avidity.

\section{Avidity ELISA}

The samples were tested using an avidity ELISA described previously [7]. The ELISA plates (Nunc MaxiSorp ${ }^{\text {TM }}$ flat bottom) were coated with $50 \mu \mathrm{l}$ of FMDV 0 antigen. The next day, plates were washed three times with blocking buffer. $50 \mu$ diluted serum sample was added in each well. Plates were kept at $37^{\circ} \mathrm{C}$ for one hour followed by three times washing by blocking buffer. The antigen and antibody complex was treated with a chaotropic agent $(7 \mathrm{M}$ Urea) in PBS for 20 minutes added $50 \mu \mathrm{l} /$ well. The samples were tested in two rows, one treated with a chaotropic agent and the other one non-treated. The plates were incubated for one hour at $37^{\circ} \mathrm{C}$ and then washed three times with PBS. Subsequently, diluted anti-bovine IgG conjugated HRPO (Sigma) (as per the manufacturer's recommended concentration) was added to the plates and kept for one hour at $37^{\circ} \mathrm{C}$ followed by three times washing with PBS + $1 \%$ Normal Rabbit Sera $+0.2 \%$ Tween 20. In the end, chromogen/ substrate mixture $(50 \mu \mathrm{l} /$ well) containing $5.05 \mathrm{mM}$ ortho-phenylene-diamine dihydrochloride (Sigma) 30\% (w/w) hydrogen perox- ide (Sigma) diluted 1:2,000 was added and the color reaction was read at $490 \mathrm{~nm}$ in an ELISA plate reader. The test was interpreted as an avidity index.

Avidity Index= Optical density (OD) of Urea treated samples X 100

OD of Urea untreated samples

\section{Cut-off value of avidity ELISA}

The cut-off value of avidity ELISA was calculated by measuring the average avidity index of the 100 negative samples. The average value +2 SD was taken as the cut-off value. Samples having avidity index more than the cut-off value were considered as having high avidity antibodies and less than the cut-off were considered as having low avidity antibodies.

\section{Statistical analysis}

The avidity indices of naïve animals, single vaccinated and multiple vaccinated animals was compared by Student's t-Test using Graph Pad Prism Software.

\section{Results}

The cut-off value was fixed in OD $=0.54$ (mean OD value of 100 negative samples $+2 \mathrm{SD}$ ). Only untreated samples (PBS wash) with an OD value over 0.54 (corresponding to LPB-ELISA titer over 1.4) were considered to calculate the AI.

\section{Cut-off value of avidity ELISA}

100 negative samples were subjected to avidity ELISA and their avidity index was calculated. The mean avidity index was 25.96 with a standard deviation of 6.40 . The cut-off value calculated was Mean +2 SD which turned out as 38.77 .

\section{Avidity Index of single and multiple vaccinated animals}

Total of forty-two serum samples from single vaccinated animals were tested out of which twenty-one serum samples were positive in indirect ELISA. Out of twenty-one serum samples, fifteen samples showed high avidity index ( $>$ 38.77) ranging from 40.84 to 58.80 which formed $35.71 \%$ of total samples and remaining six samples had low avidity ranging from 7.5 to $38.13 \%$. In multiple vaccinated animals, out of 47 serum samples, 38 samples were positive in indirect ELISA. In multiple vaccinated animals, twenty-four animals had high avidity which formed $51.06 \%$ of total samples and sixteen had low avidity which formed $34.04 \%$ of total samples (Table 1). 


\begin{tabular}{|l|c|c|c|c|c|}
\hline Vaccination status & Total samples & High Avidity & Percentage & Low avidity & Percentage \\
\hline Single vaccinated & 42 & 15 & 35.71 & 6 & 14.28 \\
\hline Multiple vaccinated & 47 & 24 & 51.06 & 14 & 29.78 \\
\hline
\end{tabular}

Table 1: A comparative analysis of avidity indices obtained in single and multiple vaccinated animals against FMD.

Comparison between avidity indices of naïve animals, single vaccinated animals and multiple vaccinated animals

The mean avidity indices of naïve animals, single vaccinated animals and multiple vaccinated animals was calculated and compared by Student's t-Test in a Graph pad prism software. A significant difference was obtained between avidity indices of naïve and single vaccinated animals ( $p$ value $=0.000$ ). Similar kind of observations were obtained for the difference between avidity indices of naïve and multiple vaccinated animals ( $p$ value $=0.000$ ). However, no significant difference was obtained between the avidity indices of single and multiple vaccinated animals ( $p$ value $=0.228$ ) (Figure 1).

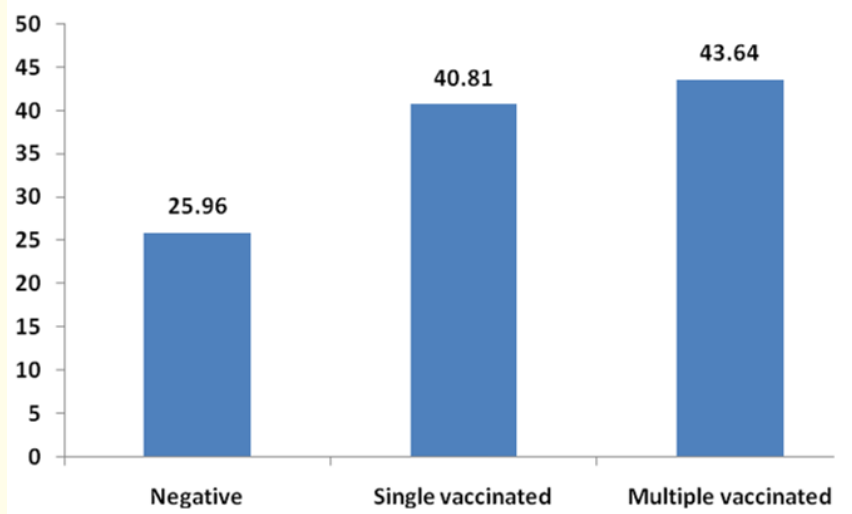

Figure 1: A comparative analysis of avidity indices of unvaccinated (negative), single vaccinated and multiple vaccinated animals. $Y$ axis indicates avidity indices.

\section{Discussion}

A strong antibody titer generated post-vaccination is suggestive of a successful vaccination regime. Further evidence of the protective nature of elicited antibodies can be confirmed by Virus neutralization test which is time-consuming. Avidity ELISA is an alternative to measure the generated immune response which in past has been used for other diseases $[2,8,9]$.
In the present study, when the qualitative analysis of antibodies generated after multiple vaccinations was done by using avidity ELISA, few serum samples were found to have antibodies with low avidity (avidity index < 38.77). Out of 47 samples tested, 16 serum samples showed low avidity with an avidity index ranging from 8.95 to 38.75 . These all 16 serum samples showed high titer ( $>1.4$ ) in LPBE. Despite a high LPBE titer, the avidity index of sixteen samples was found less $(<38.77 \%)$. Total of forty-two serum samples from single vaccinated animals were tested out of which twenty-one serum samples were positive in indirect ELISA. Therefore, multiple vaccination may not be always a true indicator of protective immune response development as many single vaccinated animals have higher avidity index than the multiple vaccinated animals. However, no significant difference was obtained in the mean avidity indices of single and multiple vaccinated animals which could be due to high avidity antibodies formed in some of the single vaccinated animals. The avidity of ELISA has serious implications in the estimation of generated immune response and hence predicting the susceptibility of that population for gaining FMDV infection. It may be helpful in predicting outbreaks where virus circulation is there but the population has low avidity index. Zones or areas having low avidity post-vaccination can be marked as having low herd immunity and continuous sero-monitoring may be done.

\section{Acknowledgments}

This work performed in this work received funding from Lala Lajpat Rai University of Veterinary and Animal Sciences, Hisar, Haryana, India.

\section{Declarations}

\section{Funding}

The work received funding from Lala Lajpat Rai University of Veterinary and Animal Sciences, Hisar, Haryana, India.

\section{Conflict of interest}

The authors declare that they have no conflict of interest. 


\section{Data Availability}

All data generated or analysed during this study are included in this published article.

\section{Code Availability}

Not applicable.

\section{Authors' Contribution}

All authors have made considerable contribution to this work. Beenu Jain conducted research, wrote first draft of manuscript and designed research. Anuj Tewari designed research, analysed data, edited manuscript. Surender Kadian supervised the work. All authors reviewed and approved the manuscript.

\section{Ethical Approval}

The study protocol was assessed and approved by Lala Lajpat Rai University of Veterinary and Animal Sciences, Hisar, India. All protocols designed by Institutional Animal Ethics Committee, Lala Lajpat Rai University of Veterinary and Animal Sciences, Hisar, India were followed while collecting serum samples from field buffaloes. Serum samples used in this study were collected by veterinarians in veterinary practices after taking prior consent from the animal owners, with minimized stress to the animal.

\section{Consent to Participate}

All authors contributed to the study conception and design. All authors read and approved the final manuscript.

\section{Consent for Publication}

All authors gave their consent for research publication.

\section{Bibliography}

1. Knight-Jones TJD and Rushton J. "The economic impacts of foot and mouth disease - What are they, how big are they and where do they occur?" Preventive Veterinary Medicine 112.3-4 (2013): 161-173.

2. Lavoria M ángeles., et al. "Avidity and subtyping of specific antibodies applied to the indirect assessment of heterologous protection against Foot-and-Mouth Disease Virus in cattle". Vaccine 30.48 (2012): 6845-6850.

3. McCullough KC., et al. "Immune protection against foot-andmouth disease virus studied using virus-neutralizing and nonneutralizing concentrations of monoclonal antibodies". Immunology. 58.3 (1986): 421-428.
4. McCullough KC., et al. "Protective immune response against foot-and-mouth disease". Journal of Virology 66.4 (1992): 1835-1840.

5. Mulcahy G., et al. "Maturation of functional antibody affinity in animals immunised with synthetic foot-and-mouth disease virus". Research in Veterinary Science 52.2(1992):133-140.

6. Scicluna LA., et al. "Qualitative assessment of the humoral immune status against FMDV in post-vaccination cattle". Vaccine 19.20-22 (2001): 2975-2986.

7. Jain B., et al. "A novel approach for estimation of anti-FMDV protective immunity generated in multiple vaccinated field animals". Acta Virologica (2022).

8. Bachmann MF., et al. "The role of antibody concentration and avidity in antiviral protection". Science 276.5321 (1997): 2024-2027.

9. Franco Mahecha OL., et al. "Single dilution Avidity-Blocking ELISA as an alternative to the Bovine Viral Diarrhea Virus neutralization test". Journal of Virological Methods 175.2 (2001): 228-235.

\section{Assets from publication with us}

- Prompt Acknowledgement after receiving the article

- Thorough Double blinded peer review

- Rapid Publication

- Issue of Publication Certificate

- High visibility of your Published work

Website: www.actascientific.com/

Submit Article: www.actascientific.com/submission.php

Email us: editor@actascientific.com

Contact us: +919182824667 